Research

Open Access

\title{
Candidemia on presentation to the hospital: development and validation of a risk score
}

\author{
Andrew F Shorr ${ }^{1}$, Ying P Tabak², Richard S Johannes ${ }^{2,3}$, Xiaowu Sun², James Spalding ${ }^{4}$ and \\ Marin $\mathrm{H}$ Kollef 5
}

\author{
${ }^{1}$ Pulmonary and Critical Care Medicine Service, Washington Hospital Center, Washington, DC 20010, USA \\ ${ }^{2}$ Clinical Research, MedMined ${ }^{\text {TM }}$ Services, CareFusion, 400 Nickerson Road, Marlborough, MA 01752, USA \\ 3Division of Gastroenterology, Harvard Medical School and Brigham and Women's Hospital, Boston, MA 02115, USA \\ ${ }^{4}$ Health Economics \& Outcomes Research, Astellas Pharma US Inc., Three Parkway North, Deerfield, IL 60015, USA \\ ${ }^{5}$ Pulmonary and Critical Care Division, Washington University School of Medicine, 660 South Euclid Ave, St. Louis, MO 63110, USA \\ Corresponding author: Andrew F Shorr, afshorr@dnamail.com
}

Received: 4 Jun 2009 Revisions requested: 7 Jul 2009 Revisions received: 26 Aug 2009 Accepted: 29 Sep 2009 Published: 29 Sep 2009

Critical Care 2009, 13:R156 (doi:10.1186/cc8110)

This article is online at: http://ccforum.com/content/13/5/R156

(C) 2009 Shorr et al.; licensee BioMed Central Ltd.

This is an open access article distributed under the terms of the Creative Commons Attribution License (http://creativecommons.org/licenses/by/2.0), which permits unrestricted use, distribution, and reproduction in any medium, provided the original work is properly cited.

\begin{abstract}
Introduction Candidemia results in substantial morbidity and mortality, especially if initial antifungal therapy is delayed or is inappropriate; however, candidemia is difficult to diagnose because of its nonspecific presentation.

Methods To develop a risk score for identifying hospitalized patients with candidemia, we performed a retrospective analysis of a large database of 176 acute-care hospitals in the United States. We studied 64,019 patients with bloodstream infection (BSI) on presentation from 2000 through 2005 (derivation cohort) and 24,685 from 2006 to 2007 (validation cohort). We used recursive partitioning (RPART) to identify the best discriminators for Candida as the cause of BSI. We compared three sets of models (equal-weight, unequal-weight, vs full model with additional variables from logistic regression model) for sensitivity analysis.
\end{abstract}

Results The RPART identified 6 variables as the best discriminators: age $<65$ years, temperature $\leq 98^{\circ} \mathrm{F}$ or severe altered mental status, cachexia, previous hospitalization within 30 days, admitted from other healthcare facility, and need for mechanical ventilation. The prevalence for patients presented with 0 through 6 risk factors in the derivation cohort was $28.7 \%$, $38.8 \%, 21.8 \%, 8.3 \%, 2.1 \%, 0.3 \%$, and $<0.1 \%$ respectively.
The corresponding candidemia rates were $0.4 \%(69 / 18,355)$, $0.8 \%(196 / 24,811), 1.6 \%(229 / 13,984), 3.2 \%(168 / 5,330)$, $4.2 \%(58 / 1,371), 9.6 \% \quad(15 / 157)$, and $27.3 \%(3 / 11)$ respectively $(P<0.0001)$. Findings were similar in the validation cohort $(P<0.0001)$. The equal-weight risk score model, which signed 1 point to each risk factor, yielded good discrimination in both cohorts with areas under the receiver operating curve (AUROCs) of 0.70 versus 0.71 (derivation versus validation). AUROC values were similar for the unequal-weight model, which signed different weight to each risk factor based on multivariable logistic regression coefficient, (AUROCs, 0.700.72). Both equal-weight and unequal-weight models were well calibrated (all Hosmer-Lemshow $P>0.10$, indicating predicted and observed candidemia rates did not differ significant across the 7 risk stratus). The full model with 16 risk factors had slightly higher AUROCs (0.74 versus 0.73 for derivation versus validation); however, 7 variables were no longer significant in the recalibrated model for the validation cohort, indicating that the additional items did not materially enhance the model.

Conclusions A simple equal-weight risk score differentiated patients' risk for candidemia in a graded fashion upon hospital presentation.

AMS: altered mental status; AUROC: area under the receiver operating curve; BSI: bloodstream infection; BUN: blood urea nitrogen; GCS: Glasgow Coma Scale; ICD-9-CM: International Classification of Diseases, Ninth Revision, Clinical Modification; NPV: negative predictive value; RPART: recursive partition. 


\section{Introduction}

Candidemia represents the fourth most common type of hospital-acquired bloodstream infection (BSI) [1-3]. More importantly, candidemia results in substantial morbidity [4-8] and mortality [7-10], especially if initial antifungal therapy is delayed or is not appropriate $[5,11,12]$. Delaying therapy by as little as 12 hours after obtaining a blood culture can double the risk of death [11]. Therefore, prompt initiation of antifungal therapy is a key determinant of outcome. Complicating efforts to identify subjects at risk for candidemia is the expansion of healthcare delivery beyond the hospital and the evolving recognition of distinct healthcare-associated infection syndromes [13-15], Candida may now represent a cause of BSI in patients presenting to the hospital [8].

Given the need to ensure appropriate and timely antifungal therapy and to optimally separate patients at low risk for candidemia from those at high risk, some form of risk stratification for candidemia becomes imperative. This is particularly true for those with candidemia on admission to the hospital because clinicians rarely consider this diagnosis in this setting. The nonspecific signs and symptoms of candidemia further frustrate efforts at early patient identification [16]. Although biomarkers such as $(1 \rightarrow 3)$ - $\beta$-D-glucan are being investigated [17], they are not likely to prove useful in patients presenting to the hospital. The traditional approach to assessing the probability of Candida as a cause of nosocomial BSI has relied upon assessing the number and type of risk factors (e.g., corticosteroid therapy, total parenteral nutrition); however, this strategy has proven to have little utility in critically ill patients and proposed schema for risk stratification have yet to be well validated.

We hypothesized that, despite frustration with clinical risk stratification paradigms for inpatients, assessment of select characteristics could identify patients presenting to the hospital who are at heightened risk for candidemia. We further theorized that these select characteristics could be used to develop a prediction rule to indicate which patients are likely to have BSI due to Candida as opposed to a bacterial pathogen.

\section{Materials and methods Design}

To develop a clinical risk score for identifying patients with BSI likely to be caused by Candida spp. upon hospital presentation, we performed a retrospective analysis of patients discharged from 176 acute-care hospitals in the United States from 2000 to 2005 . We validated the risk score with discharge data from the same hospitals from 2006 to 2007.

\section{Data}

We used the CareFusion Outcomes Research Database (Clinical Research Services, CareFusion, Marlborough, MA, USA), which has been described previously [14,15,18-22].
The database comprises acute-care admissions at participating hospitals, including electronically imported or manually abstracted demographic, clinical (e.g., comorbidities, vital signs, laboratory values, other clinical findings), and administrative data (e.g., diagnosis). The underlying data for this study are a limited data set with all patient specific information anonymized. This study was reviewed and approved by the New England Institutional Review Board/Human Subjects Research Committee (Wellesley, MA, USA). It was conducted in compliance with US federal regulations, Health Insurance Portability and Accountability Act, and the Helsinki Declaration.

The outcome for deriving the risk score was BSI due to Candida spp. as defined by the presence of a blood culture positive for Candida, and a concomitant primary or secondary diagnostic code (International Classification of Diseases, Ninth Revision, Clinical Modification (/CD-9-CM)) indicative of candidemia. We required that blood samples had been drawn within one day before or within two days after hospital admission. This database undergoes multiple quality assurance assessments with periodic data auditing. In order to limit coding bias we require concomitant presence of an $I C D-9$ code for candidemia and a positive blood culture. We did not explore other forms of invasive candidiasis.

\section{Variables}

Candidate variables were selected a priori based on their biologic plausibility of explaining risk for candidemia. Specifically, we explored demographic factors (age, gender), vital signs, mental status, laboratory test results, and underlying comorbid conditions. Vital signs included pulse, blood pressure, temperature, and respiratory rate. Altered mental status (AMS) was defined by a Glasgow Coma Scale (GCS) score of 10 to 14 or disoriented/lethargy (mild AMS); GCS 5 to 9 (moderate AMS); GCS less than 5 or a designation of 'coma' as charted by a physician (severe AMS). Laboratory testing included serum albumin; blood urea nitrogen (BUN); creatinine; sodium; potassium; glucose; hemoglobin; white blood cell count; and other routine chemistry, hematology, blood gas, and metabolic results. Comorbid conditions included cachexia (ICD-9 secondary diagnosis code), history of malignancy, diabetes, chronic heart failure, and other chronic conditions abstracted through chart review or secondary $I C D-9$ diagnostic codes. In addition, we explored variables pertinent to candidemia and were available in the data base, such as hemodialysis, immunosuppressive medication, previous hospitalization within 30 days, transfer from another healthcare facility, and mechanical ventilation on admission. Certain patient characteristics were not available in this database. For example, utilization of parenteral nutrition outside the hospital and prior antibiotic exposure are not recorded in this database. Vital signs and other patient-specific characteristics were obtained within one day of admission. For each vital sign and laboratory test result, we used the worst value obtained in the 
emergency department or, if not available, on the day of admission.

\section{Risk score development}

To identify risk factors that optimally separate patients at low risk for candidemia from those at high risk, we used a recursive partition (RPART) approach [23]. Also referred to as classification and regression tree analysis [24], RPART has been used to derive prediction rules for acute chest pain [25], heart failure [26], and other conditions [27,28]. RPART first identifies the variable with the highest discrimination for the outcome of interest (node) and then repeats the process to partition subsequent nodes. RPART yields a tree-like algorithm with numerous nodes. To further improve ease of use, we simplified the algorithm based on the number of risk factors present, giving equal weight (one point) to each risk factor identified in by the RPART (equal-weight risk score).

\section{Risk score validation}

To validate the model, we applied the derived risk score to patients in the validation cohort. We compared the betweencohort distribution of candidemia prevalence by risk score strata for the validation cohort with that from the derivation cohort and performed the Cochrane-Armitage test to assess trend [29]. We used the area under the receiver operating curve (AUROC) to assess the discrimination of the model and Hosmer-Lemshow test to assess model calibration. A higher value for the Hosmer-Lemshow test indicates better model fit.

\section{Sensitivity analysis}

Using AUROC and Hosmer-Lemshow goodness-of-fit statistics, we compared the discrimination and calibration of the simpler versus more complex models. Specifically, we fit three sets of logistic regression models. The first was the equalweight risk-score model, which was a univariate logistic regression model using a single continuous variable of the number of risk factors present (ranging from 0 to 6 ). This model gave the same weight for each risk factor present. The second was the unequal-weight risk factor model, which was a multivariable logistic regression model using each of the same variables in the equal-weight risk score as covariates. The unequal weight model assigned different weights for each variable per multivariable logistic regression coefficients. The third model was the full risk factor model, which was generated from a stepwise multivariable logistic regression analysis with additional variables retained in the model that were significant $(P<0.05)$.

Statistical analyses were performed using Statistical Analysis Software (SAS, version 9.01; SAS Institute Inc., Cary, NC, USA). Two-sided $P$ values $<0.05$ were considered statistically significant.

\section{Results \\ Baseline characteristics of derivation and validation cohorts}

The derivation cohort included 64,019 admissions and the validation cohort included 24,685 (Table 1) [see Additional data file 1]. Many between-cohort differences in demographics, laboratory findings, vital signs, comorbidities, and other variables were statistically significant. For example, the derivation cohort had a smaller proportion of patients aged 64 years or younger, smaller proportion of men, and higher in-hospital mortality. Approximately $10 \%$ of patients needed mechanical ventilation on admission, including $9.2 \%$ of those in the derivation cohort and $10.9 \%$ of those in the validation cohort. Among patients needing mechanical ventilation, candidemia occurred in $2.3 \%$ of those in the derivation cohort and in $3.1 \%$ of those in the validation cohort (Table 2) [see Additional data file 2].

\section{Derivation and validation of candidemia risk score}

Univariate analysis revealed that the following variables were associated with candidemia: age younger than 65 years; cachexia; deranged albumin, arterial $\mathrm{pH}$, and electrolytes; temperature of $98^{\circ} \mathrm{F}$ or less, or severe altered mental status; previous hospitalization within 30 days; admitted from other healthcare facility; and mechanical ventilation at admission (all $P \leq 0.001$; Table 2). These associations were similar in the derivation and validation cohorts.

RPART revealed that the six best discriminators for candidemia were age younger than 65 years, temperature of $98^{\circ} \mathrm{F}$ or less, or severe altered mental status, cachexia, previous hospitalization within 30 days, admitted from other healthcare facility, and mechanical ventilation at admission. The prevalence for patients presented with 0 through to 6 risk factors in the derivation cohort was $28.7 \%, 38.8 \%, 21.8 \%, 8.3 \%, 2.1 \%$, $0.3 \%$, and less than $0.1 \%$, respectively. The corresponding candidemia rates were $0.4 \%(69 / 18,355), 0.8 \% \quad(196 /$ 24,811), 1.6\% (229/13,984), 3.2\% (168/5330), 4.2\% (58/ 1371), 9.6\% (15/157), and 27.3\% (3/11), respectively $(P<$ $0.0001)$. Findings were similar in the validation cohort $(P<$ 0.0001 ; Figure 1). The Cochrane-Armitage test for trend was significant $(P<0.0001)$, confirming graded risk of candidemia with increased number of risk factors. Findings were similar in the validation cohort. The equal weight risk-score model provided good discrimination as demonstrated by the AUROC of 0.70 for the derivation cohort and 0.71 for the validation cohort (Figure 2).

In the derivation cohort, an overall score of 1 or more had an sensitivity of $90.7 \%$ and a negative predictive value (NPV) of $99.6 \%$ for the presence of candidemia. The specificity was more limited at $28.9 \%$. The negative predictive value of each total point score remained above $99 \%$ so long as the number of risk factors presented remained less than 3 . These findings were similar in the validation cohort. In other words, a low score nearly excluded the likelihood of candidemia. In patients 
Number of admissions (\% of total)

\begin{tabular}{|c|c|c|c|}
\hline Characteristic & Derivation cohort $(n=64,019)$ & Validation cohort $(n=24,685)$ & $P$ value \\
\hline Candidemia & $738(1.2)$ & $321(1.3)$ & 0.0697 \\
\hline \multicolumn{4}{|l|}{ Demographics } \\
\hline Age $<65$ years & $19,523(30.5)$ & $8403(34.0)$ & $<0.0001$ \\
\hline Men & $29,845(46.6)$ & $12,090(49.0)$ & $<0.0001$ \\
\hline Mortality & $9664(15.1)$ & $3173(12.9)$ & $<0.0001$ \\
\hline \multicolumn{4}{|l|}{ Laboratory findings } \\
\hline Albumin $\leq 1.8 \mathrm{~g} / \mathrm{dL}$ & $2704(4.2)$ & $1278(5.2)$ & $<0.0001$ \\
\hline Albumin $1.9--2.2 \mathrm{~g} / \mathrm{dL}$ & $3800(5.9)$ & $1757(7.1)$ & $<0.0001$ \\
\hline Arterial $\mathrm{pH} \leq 7.36$ & $5913(9.2)$ & $2684(10.9)$ & $<0.0001$ \\
\hline Potassium > $>5.6 \mathrm{mEq} / \mathrm{dL}$ & $3133(4.9)$ & $1182(4.8)$ & 0.5150 \\
\hline Sodium > $145 \mathrm{mEq} / \mathrm{dL}$ & $3600(5.6)$ & $1206(4.9)$ & $<0.0001$ \\
\hline Bands $>32 \%$ & $5345(8.4)$ & $1813(7.4)$ & $<0.0001$ \\
\hline White blood cells $>27,000 / \mathrm{mm}^{3}$ & $5096(8.0)$ & $1980(8.0)$ & 0.7604 \\
\hline \multicolumn{4}{|l|}{ Vital signs and mental status } \\
\hline Temperature $\leq 98^{\circ} \mathrm{F}$ & $16,917(26.4)$ & $4780(19.4)$ & $<0.0001$ \\
\hline Severe altered mental status ${ }^{a}$ & $6301(9.8)$ & $2255(9.1)$ & 0.0014 \\
\hline Temperature $\leq 98^{\circ} \mathrm{F}$ or severe altered mental status & $21,140(33.0)$ & $6454(26.1)$ & $<0.0001$ \\
\hline \multicolumn{4}{|l|}{ History and severe comorbidities } \\
\hline Metastatic cancer & $3245(5.1)$ & $1262(5.1)$ & 0.7910 \\
\hline Tumor & $2693(4.2)$ & $1136(4.6)$ & 0.0094 \\
\hline Cachexia ${ }^{b}$ & $4549(7.1)$ & $2392(9.7)$ & $<0.0001$ \\
\hline \multicolumn{4}{|l|}{ Other variables } \\
\hline Prior-admission within 30 days & $11,215(17.5)$ & $4603(18.7)$ & $<0.0001$ \\
\hline Admitted from other healthcare facility & $12,813(20.0)$ & $5581(22.6)$ & $<0.0001$ \\
\hline Mechanical ventilation at admission & $5864(9.2)$ & $2695(10.9)$ & $<0.0001$ \\
\hline
\end{tabular}

a Severe altered mental status defined as Glasgow Coma Scale less than 5 or a designation of coma by a physician.

${ }^{b}$ Cachexia defined by $I C D-9$ secondary diagnosis code.

with a score of zero, who account for nearly $30 \%$ of all subjects evaluated, there were very few cases of candidemia, with a NPV of $99.6 \%$.

\section{Sensitivity analysis}

The equal-weight risk model was associated with discrimination similar to that of the unequal-weight model (Table 3). The AUROCs (95\% confidence intervals) for the equal-weight riskscore model were 0.70 ( 0.68 to 0.72$)$ for the derivation cohort and 0.71 ( 0.68 to 0.74$)$ for the validation cohort. The corresponding values for the unequal-weight model were 0.71 (0.70 to 0.73 ) and 0.72 (0.69 to 0.75 ). The full model with 16 risk factors was associated with slightly higher discrimination in both cohorts, with corresponding values of 0.74 (0.72 to 0.76 ) and 0.73 (0.70 to 0.76 ). Seven variables in 16-risk factor model, however, were not significant in the recalibrated model for the validation cohort, suggesting that using the additional covariates did not materially enhance the model.

Both the equal and unequal weight models provided good calibration of predicted versus observed candidemia across lowand high-risk strata as demonstrated by insignificant $P$ values in both cohorts (all Hosmer-Lemshow chi-squared test $P>$ 0.10 , a larger $P$ value is better, because it suggests that predicted and observed incident rates are in higher agreement across low and high risk stratus). The full model also provided 
Univariate analysis of variables associated with candidemia

\begin{tabular}{|c|c|c|c|c|}
\hline \multirow[b]{2}{*}{ Variable } & \multicolumn{2}{|l|}{ Derivation cohort $(n=64,019)$} & \multicolumn{2}{|l|}{ Validation cohort $(n=24,685)$} \\
\hline & $\begin{array}{c}\text { Number of candidemia/Number of } \\
\text { cases in the row (\%) }\end{array}$ & $P$ Value & $\begin{array}{c}\text { Number of candidemia/Number of } \\
\text { cases in the row (\%) }\end{array}$ & $P$ Value \\
\hline Candidemia cases/Total cases & $738 / 64,019(1.2)$ & & $321 / 24,685$ (1.3) & 0.0697 \\
\hline \multicolumn{5}{|l|}{ Demographics } \\
\hline Age $<65$ years & $331 / 19,523(1.7)$ & $<0.0001$ & $137 / 8403(1.6)$ & 0.0010 \\
\hline Men & $377 / 29,845$ (1.3) & 0.0158 & $170 / 12,090(1.4)$ & 0.1508 \\
\hline \multicolumn{5}{|l|}{ Laboratory findings } \\
\hline Albumin $\leq 1.8 \mathrm{~g} / \mathrm{dL}$ & $83 / 2704(3.1)$ & $<0.0001$ & $40 / 1278(3.1)$ & $<0.0001$ \\
\hline Albumin $1.9--2.2 \mathrm{~g} / \mathrm{dL}$ & $75 / 3800(2.0)$ & $<0.0001$ & $45 / 1757(2.6)$ & $<0.0001$ \\
\hline Arterial $\mathrm{pH} \leq 7.36$ & $130 / 5913(2.2)$ & $<0.0001$ & $61 / 2684(2.3)$ & $<0.0001$ \\
\hline Potassium $>5.6 \mathrm{mEq} / \mathrm{dL}$ & $80 / 3133(2.6)$ & $<0.0001$ & $34 / 1182(2.9)$ & $<0.0001$ \\
\hline Sodium $>145 \mathrm{mEq} / \mathrm{dL}$ & $74 / 3600(2.1)$ & $<0.0001$ & $34 / 1206(2.8)$ & $<0.0001$ \\
\hline Bands $>32 \%$ & $50 / 5345(0.9)$ & 0.1239 & $26 / 1813(1.4)$ & 0.6021 \\
\hline White blood cells $>27,000 / \mathrm{mm}^{3}$ & $54 / 5096(1.1)$ & 0.5840 & $35 / 1980(1.8)$ & 0.0557 \\
\hline \multicolumn{5}{|l|}{ Vital signs and mental status } \\
\hline Temperature $\leq 98^{\circ} \mathrm{F}$ & $253 / 16,917$ (1.5) & $<0.0001$ & $99 / 4780(2.1)$ & $<0.0001$ \\
\hline Severe altered mental status ${ }^{a}$ & $135 / 6301(2.1)$ & $<0.0001$ & $51 / 2255(2.3)$ & $<0.0001$ \\
\hline $\begin{array}{l}\text { Temperature } \leq 98^{\circ} \mathrm{F} \text { or severe } \\
\text { altered mental status }\end{array}$ & $336 / 21,140(1.6)$ & $<0.0001$ & $129 / 6454(2.0)$ & $<0.0001$ \\
\hline \multicolumn{5}{|l|}{ History and severe comorbidities } \\
\hline Metastatic cancer & $62 / 3245(1.9)$ & 0.0001 & $25 / 1262(2.0)$ & 0.0285 \\
\hline Tumor & $41 / 2693(1.5)$ & 0.0786 & $19 / 1136(1.7)$ & 0.2570 \\
\hline Cachexia $^{b}$ & $123 / 4549(2.7)$ & $<0.0001$ & $70 / 2392(2.9)$ & $<0.0001$ \\
\hline \multicolumn{5}{|l|}{ Other variables } \\
\hline Pre-admission within 30 days & $276 / 11,215(2.5)$ & $<0.0001$ & $125 / 4603(2.7)$ & $<0.0001$ \\
\hline $\begin{array}{l}\text { Admitted from other healthcare } \\
\text { facility }\end{array}$ & $281 / 12,813(2.2)$ & $<0.0001$ & $133 / 5581(2.4)$ & $<0.0001$ \\
\hline $\begin{array}{l}\text { Mechanical ventilation at } \\
\text { admission }\end{array}$ & $136 / 5864(2.3)$ & $<0.0001$ & $84 / 2695(3.1)$ & $<0.0001$ \\
\hline
\end{tabular}

a Severe altered mental status defined as Glasgow Coma Scale less than 5 or a designation of coma by a physician.

${ }^{b}$ Cachexia defined by $I C D-9$ secondary diagnosis code.

good calibration in the derivation cohort $(P=0.74)$ but not in the validation cohort $(P=0.02)$, suggesting over- or underprediction in some risk strata when additional variables were added to the model.

\section{Discussion}

Our analysis demonstrates that a simple equal-weight risk stratification score can assess the potential for candidemia in newly hospitalized patients with BSI. We validated our model using a cohort of patients discharged during the two consecutive years after the derivation cohort. The cohorts had similar graded risk of candidemia that increased with increased number of risk factors. The equal-weight risk-score model provided similar between-cohort discrimination for the risk of candidemia and goodness of model fit, indicating the stability of our risk score. In a sensitivity analysis, the equal-weight riskscore model provided nearly identical discrimination and goodness of fit compared with that of unequal-weight model. A full 16-risk factor model provided slightly better discrimination but was less robust. Importantly, the equal-weight riskscore model is easier to apply than the other two models. 
Figure 1

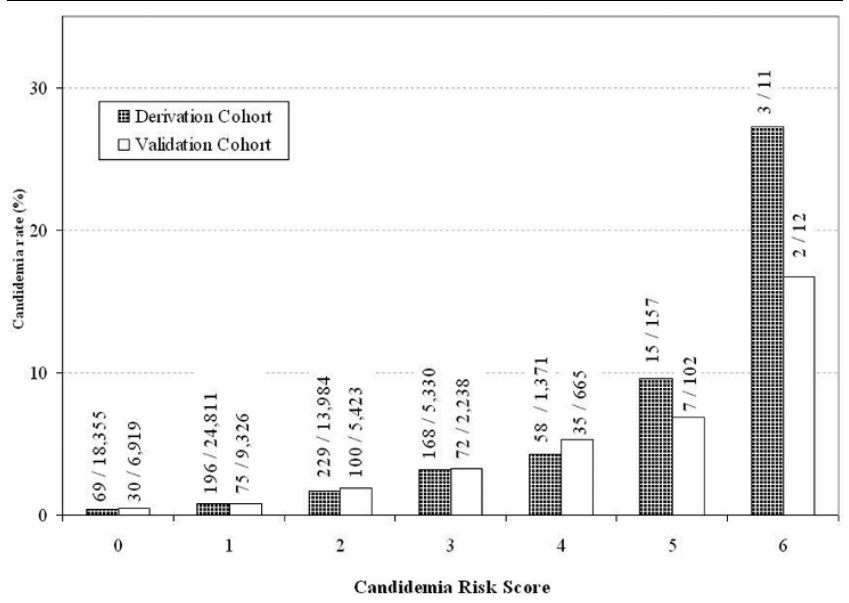

Distribution of overall cases and Candidemia cases by the equal-weight Candidemia Risk Score.

The need for a risk stratification scheme is pressing. Although Candida may be an infrequent cause of BSI on admission, epidemiologic data indicate that the rate of this is likely to increase. The expansion of healthcare delivery beyond the hospital continues apace, and multiple studies now document the evolution of healthcare-associated infections that are distinct from community-acquired or nosocomial infections [1315]. The likelihood of an increasing prevalence of candidemia at admission, along with the need to ensure that such patients receive early and appropriate antifungal therapy, underscores the anticipated benefit of easy-to-use risk stratification. Prior efforts at risk stratification for candidemia as a cause of nosocomial BSI have been largely unsuccessful due to the lack of a large clinical data set to model such infrequent events. Our

\section{Figure 2}

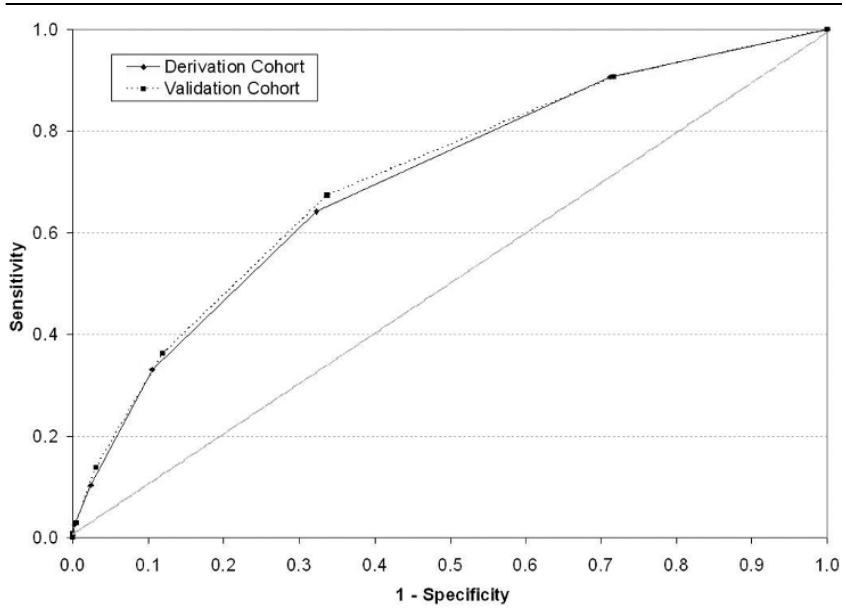

Receiver operating characteristics curves for the equal-weight Candidemia Risk Score by cohort. The area under the receiver operating curve was 0.70 for the derivation cohort and 0.71 for the validation cohort. effort builds on earlier analyses $[30,31]$ by focusing on a distinct cohort of patients and by using multiple statistical methods to cross-validate the algorithms. Moreover, many adjuncts to a clinically based risk stratification scheme, such as relying on the colonization index or serodiagnostic testing, are less likely to be available in patients presenting to the hospital.

Our risk-score comprised six demographic, patient history, and clinical findings that are routinely available in any acutecare hospital setting and that were previously shown to be associated with adverse outcomes $[8,32]$. To minimize the time needed to assess the risk of candidemia, we excluded variables requiring laboratory testing.

Our risk score offers several advantages over previous models $[30,31]$. First, as noted above, our variables were routinely available at presentation and did not require cultures or other tests to confirm the presence of colonization, sepsis, or other conditions. This increases the scores practical value for rapid assessment of risk for candidemia. Second, the accuracy and robustness of our risk score was supported by derivation from a cohort comprising 64,019 patients and validation from a different cohort comprising 24,685 patients in a different time period. Most previous studies of risk assessment in candidemia did not include any retrospective or prospective validation. Third, our results are likely to be generalizable to a broad range of patients presenting to acute-care hospitals because they are derived from teaching and non-teaching hospitals and from urban and rural hospitals, and are not limited to patients in intensive care units. Fourth, we used the concomitant presence of candidemia code and a positive blood culture to identify candidemia case and included acute clinical presentation on admission as candidate variables, which is likely to be a strength of our paper because many large-scale databases tend to only have the results of administrative coding and lack actual culture confirmation.

Our risk score seems consistent with the pathogenesis of candidemia, which includes: increased fungal burden or colonization, often due to broad-spectrum antibacterial therapy or previous health care exposure; disruption of mucosal and skin barriers, often due to indwelling vascular catheters, surgery, trauma, or chemotherapy-related mucositis; and immune dysfunction, which allows dissemination of fungal colonies [16]. For example, previous admission within 30 days and admission from another health care facility, which were important in our model, are likely represent markers for the first and second steps in the pathogenesis of candidemia. Secondly, the relationship between the need for mechanical ventilation and candidemia has been confirmed by others [32]. Although previous studies found that age was not an independent risk factor for candidemia [30,33], our analyses revealed that among patients with BSls the younger ones appear potentially more iatrogenically immunosuppressed. For example, patients aged less than 65 years were more likely to be on immunosuppres- 


\begin{tabular}{|c|c|c|c|c|}
\hline \multirow[b]{2}{*}{ Variable } & \multicolumn{2}{|c|}{ Derivation cohort } & \multicolumn{2}{|c|}{ Validation cohort } \\
\hline & OR $(95 \% \mathrm{Cl})$ & $P$ value $a$ & OR $(95 \% \mathrm{Cl})$ & $P$ value \\
\hline Equal weight risk-score model & \multicolumn{2}{|c|}{$\mathrm{AUROC}^{b}=0.70 ; \mathrm{H}-\mathrm{L} P=0.39$} & \multicolumn{2}{|c|}{ AUROC $=0.71 ; \mathrm{H}-\mathrm{L} P=0.34$} \\
\hline Number of risk factors present (0--6) & $1.93(1.81--2.04)$ & $<0.0001$ & $1.89(1.73--2.06)$ & $<0.0001$ \\
\hline Unequal weight risk model & \multicolumn{2}{|c|}{ AUROC $=0.71 ; \mathrm{H}-\mathrm{L} P=0.47$} & \multicolumn{2}{|c|}{ AUROC $=0.72 ; \mathrm{H}-\mathrm{L} P=0.66$} \\
\hline Age $<65$ years & $2.08(1.79--2.41)$ & $<0.0001$ & $1.53(1.21--1.92)$ & 0.0003 \\
\hline Temperature $\leq 98^{\circ} \mathrm{F}$ or severe altered mental status ${ }^{c}$ & $1.43(1.23--1.66)$ & $<0.0001$ & $1.43(1.13--1.81)$ & 0.0030 \\
\hline Cachexia $^{d}$ & $2.16(1.77--2.64)$ & $<0.0001$ & $2.01(1.53--2.65)$ & $<0.0001$ \\
\hline Prior admission within 30 days & $2.54(2.18--2.96)$ & $<0.0001$ & $2.50(1.99--3.15)$ & $<0.0001$ \\
\hline Admitted from other health care facility & $2.28(1.95--2.66)$ & $<0.0001$ & $2.06(1.63--2.60)$ & $<0.0001$ \\
\hline Mechanical ventilation at admission & $1.56(1.28--1.90)$ & $<0.0001$ & $2.07(1.58--2.71)$ & $<0.0001$ \\
\hline Full risk model (16 risk factors) & \multicolumn{2}{|c|}{ AUROC $=0.74 ; \mathrm{H}-\mathrm{L} P=0.02$} & \multicolumn{2}{|c|}{ AUROC $=0.73 ; \mathrm{H}-\mathrm{L} P=0.74$} \\
\hline Age $<65$ years & $2.03(1.75--2.37)$ & $<0.0001$ & $1.52(1.21--1.92)$ & 0.0004 \\
\hline Admitted from other health care facility & $2.27(1.93--2.65)$ & $<0.0001$ & $2.06(1.63--2.61)$ & $<0.0001$ \\
\hline Mechanical ventilation at admission & $1.28(1.02--1.61)$ & 0.0368 & $2.03(1.48--2.78)$ & $<0.0001$ \\
\hline Metastatic cancer & $1.57(1.20--2.05)$ & 0.0011 & $1.43(0.94--2.18)$ & 0.0994 \\
\hline Tumor & $1.47(1.06--2.02)$ & 0.0202 & $1.36(0.85--2.19)$ & 0.2008 \\
\hline Cachexia & $1.99(1.62--2.43)$ & $<0.0001$ & $1.82(1.38--2.41)$ & $<0.0001$ \\
\hline Pre-admission within 30 days & $2.41(2.07--2.81)$ & $<0.0001$ & $2.35(1.86--2.97)$ & $<0.0001$ \\
\hline Albumin $\leq 1.8 \mathrm{~g} / \mathrm{dL}$ & $1.70(1.33--2.18)$ & $<0.0001$ & $1.48(1.03--2.13)$ & 0.0329 \\
\hline Albumin $1.9--2.2 \mathrm{~g} / \mathrm{dL}$ & $1.35(1.05--1.73)$ & 0.0184 & $1.59(1.14--2.22)$ & 0.0061 \\
\hline Potassium $>5.6 \mathrm{mEq} / \mathrm{dL}$ & $1.61(1.26--2.06)$ & 0.0002 & $1.42(0.97--2.08)$ & 0.0747 \\
\hline Sodium $>145 \mathrm{mEq} / \mathrm{dL}$ & $1.30(1.00--1.68)$ & 0.047 & $1.51(1.03--2.20)$ & 0.0345 \\
\hline Arterial $\mathrm{pH}<7.36$ & $1.38(1.10--1.74)$ & 0.0058 & $1.00(0.71--1.41)$ & 0.9896 \\
\hline Bands $>32 \%$ & $0.66(0.49--0.88)$ & 0.0051 & $0.87(0.57--1.31)$ & 0.4991 \\
\hline White blood cells $>27,000 / \mathrm{mm}^{3}$ & $0.69(0.52--0.92)$ & 0.0105 & $1.09(0.76--1.57)$ & 0.6296 \\
\hline Temperature $\leq 98^{\circ} \mathrm{F}$ & $1.22(1.04--1.43)$ & 0.0130 & $1.52(1.19--1.95)$ & 0.0009 \\
\hline Severe altered mental status & $1.26(1.01--1.56)$ & 0.0388 & $0.94(0.67--1.33)$ & 0.7403 \\
\hline
\end{tabular}

AUROC $=$ area under receiver operating curve; $\mathrm{Cl}=$ confidence interval; $\mathrm{H}-\mathrm{L}=$ Hosmer-Lemshow chi-squared test; OR $=$ odds ratio.

a $\mathrm{H}-\mathrm{L} P$ values for the three models were determined by Hosmer-Lemshow chi-squared test in which $P>0.05$ indicates good model calibration of predicted vs observed candidemia across low- and high-risk deciles. $P$ values for each variable within the models were determined by each logistic regression model in which a significant value indicates increased risk for candidemia.

$b$ AUROC $\geq 0.70$ indicates good model discrimination.

${ }^{c}$ Severe altered mental status defined by Glasgow Coma Scale less than 5 or a designation of coma by a physician.

${ }^{d}$ Cachexia defined by $I C D-9$ secondary diagnosis code.

sive therapy (17.0\% versus $12.6 \% ; P<0.0001)$, hemodialysis (4.5\% versus $2.7 \%, P<0.0001$ ), or have metastatic cancer (6.0\% versus $4.7 \% ; P<0.0001)$. Similarly, cachexia was associated with metastatic cancer $(6.7 \%$ versus $4.9 \%$; $P<$ 0.0001 ), immunosuppressed status, or other severe clinical conditions making patients prone to repeated hospitalization and infections. Furthermore, hypothermia is a risk factor for greater mortality with infection and may suggest that fungal infections are often more severe when detected, or more likely to have a delay in therapy resulting in hypothermia and potentially worse outcomes [34]. In total, our risk score probably captured composite measures for exposures to healthcare delivery and its associated risks for candidemia such as underlying immunosuppression and severity of illness -- both expected risk factors for candidemia. Hence the model appeared robust overall when applied to a separate patient 
population in a different time period in the validation cohort. The high NPV of a low score indicates that the clinical value of the equal-weight score lies in its ability to identify a group of patients at an exceedingly low risk for candidemia. Given an overall prevalence of $1.2 \%$, which essentially represents the pre-test probability of candidemia in these patients, application of the risk score selects for a group of patients where the risk of candidemia approximates zero. In these subjects antifungal therapy can likely be withheld safely because a low score essentially rules out candidemia. More importantly, this very-low-risk group comprises the bulk of the subjects. Alternatively, although the prevalence of candidemia in the higherrisk groups remains limited, the score at least can serve to remind clinicians to consider candidemia and to weigh the potential for this along with the presence or absence of other clinical factors.

Our model had several limitations. First, the retrospective design needs to be validated in a prospective study. However, only large databases provide a sufficiently large sample to identify enough candidemia cases for multivariable modeling. To address issues related to bias from utilization of $I C D-9$ coding, we required culture evidence of candidemia. Second, we limited our population to patients with candidemia diagnosed within two days of admission. Extending the observation period may have changed our model. Therefore, our findings are not necessarily applicable for suspected nosocomial candidemia. Similarly, we likely missed cases present at admission but not diagnosed until later during hospitalization because cultures are not always obtained upon admission. Third, information was lacking on some specific risk factors for candidemia. For example, we did not have data on whether patients were receiving total parenteral nutrition on admission, had central venous catheters in place, had been exposed to antimicrobial therapy, or had recently undergone surgery $[30,31,33,35]$. Nevertheless, we included previous hospitalization within 30 days, immunosuppression status, and cachexia as candidate variables, which were likely to be associated with those known risk factors identified in the previous literature. Our score is meant to serve as an adjunct to clinical decision-making, which might incorporate knowledge of all potential risk factors. It is not meant in any way to supplant bedside decision-making. Finally, our analysis focused on subjects presenting to the hospital. Therefore, this score does not necessarily apply in cases of suspected nosocomial candidemia.

\section{Conclusions}

In conclusion, we derived and validated a simple risk-score model that stratifies patients at risk for candidemia, which may help clinicians to rule out candidemia and to shorten the time required to identify patients at increased risk for this disease. It may also help researchers to stratify clinical trial or other outcome studies based on the risk present. Although prospective validation is required, six easy-to-determine characteristics categorize candidemia risks at early hospitalization.

\section{Key messages}

- Candidemia is associated with substantial morbidity and mortality, yet it is difficult to diagnose because of its nonspecific presentation.

- We developed and validated a risk score consisting of six easy-to-determine characteristics on presentation.

- The candidemia risk score differentiates patients from low to high risk in a graded fashion.

- The risk score may aid physicians in ruling out candidemia and in identifying those at high risk for candidemia early in the hospital stay. It may also be useful for stratifying patients in clinical trials or other outcome studies.

\section{Competing interests}

AFS and MHK have received grant support from, and served as investigators for and consultants to Astellas Pharma US, Inc., Merck, and Pfizer. YPT, XS, and RSJ are employees of CareFusion. JS is an employee of Astellas Pharma US, Inc. Acknowledged contributors Vikas Gupta, Ed Cox, and Linda Hyde are employees of Cardinal Health.

\section{Authors' contributions}

AFS contributed to study concept and design, analysis and interpretation of data, drafting the manuscript, critical revision of the manuscript for important intellectual content, statistical expertise, obtained funding and study supervision.

YPT contributed to study concept and design, acquisition of data, analysis and interpretation of data, drafting the manuscript, critical revision of the manuscript for important intellectual content, statistical expertise, obtained funding, administrative, technical, or material support and study supervision.

RSJ contributed to study concept and design, acquisition of data, analysis and interpretation of data, drafting the manuscript, critical revision of the manuscript for important intellectual content, statistical expertise, and administrative, technical, or material support

XS contributed to study concept and design, acquisition of data, analysis and interpretation of data, drafting the manuscript, critical revision of the manuscript for important intellectual content, statistical expertise and administrative, technical, or material support.

JS contributed to study concept and design, drafting the manuscript, critical revision of the manuscript for important intellectual content, and obtained funding.

MHK contributed to study concept and design, analysis and interpretation of data, and critical revision of the manuscript for important intellectual content. 


\section{Additional files}

The following Additional files are available online:

\section{Additional file 1}

Word file containing a table that lists the detailed patient characteristics by derivation and validation cohort.

See http://www.biomedcentral.com/content/

supplementary/cc8110-S1.DOC

\section{Additional file 2}

Word file containing a table that lists detailed univariate analysis on variables associated with candidemia. See http://www.biomedcentral.com/content/ supplementary/cc8110-S2.DOC

\section{Acknowledgements}

We thank the following staff members of CareFusion Clinical Research Services for their dedicated contributions in obtaining funds, database management, analysis, and technical support: Vikas Gupta, Ed Cox, and Linda Hyde. We thank Cindy W. Hamilton of Hamilton House, Virginia Beach, VA, for providing medical writing and editing services; Hamilton House received payment from CareFusion Clinical Research Services for its services.

\section{References}

1. Pfaller MA, Jones RN, Messer SA, Edmond MB, Wenzel RP: National surveillance of nosocomial blood stream infection due to Candida albicans: frequency of occurrence and antifungal susceptibility in the SCOPE Program. Diagn Microbiol Infect Dis 1998, 31:327-332.

2. Richards MJ, Edwards JR, Culver DH, Gaynes RP: Nosocomial infections in combined medical-surgical intensive care units in the United States. Infect Control Hosp Epidemiol 2000, 21:510-515.

3. Wisplinghoff $H$, Bischoff $T$, Tallent SM, Seifert H, Wenzel RP, Edmond MB: Nosocomial bloodstream infections in US hospitals: analysis of 24,179 cases from a prospective nationwide surveillance study. Clin Infect Dis 2004, 39:309-317.

4. Gagne JJ, Breitbart RE, Maio V, Horn DL, Hartmann CW, Swanson $\mathrm{R}$, Goldfarb NI: Costs associated with candidemia in a hospital setting. P\&T 2006, 31:586-619.

5. Morgan J, Meltzer MI, Plikaytis BD, Sofair AN, Huie-White S, Wilcox S, Harrison LH, Seaberg EC, Hajjeh RA, Teutsch SM: Excess mortality, hospital stay, and cost due to candidemia: a casecontrol study using data from population-based candidemia surveillance. Infect Control Hosp Epidemio/ 2005, 26:540-547.

6. Rentz AM, Halpern MT, Bowden R: The impact of candidemia on length of hospital stay, outcome, and overall cost of illness. Clin Infect Dis 1998, 27:781-788.

7. Puzniak L, Teutsch S, Powderly W, Polish L: Has the epidemiology of nosocomial candidemia changed? Infect Control Hosp Epidemiol 2004, 25:628-633.

8. Shorr AF, Gupta V, Johannes RS, Sun X, Spalding J, Tabak YP: Burden of early-onset candidemia: analysis of culture-positive bloodstream infections from a large US database. Crit Care Med 2009, 37:2519-26.

9. Falagas ME, Apostolou KE, Pappas VD: Attributable mortality of candidemia: a systematic review of matched cohort and case- control studies. Eur J Clin Microbiol Infect Dis 2006, 25:419-425.

10. Gudlaugsson O, Gillespie S, Lee K, Berg J Vande, Hu J, Messer S, Herwaldt L, Pfaller M, Diekema D: Attributable mortality of nosocomial candidemia, revisited. Clin Infect Dis 2003, 37:1172-1177.

11. Morrell M, Fraser VJ, Kollef MH: Delaying the empiric treatment of Candida bloodstream infection until positive blood culture results are obtained: a potential risk factor for hospital mortality. Antimicrob Agents Chemother 2005, 49:3640-3645.

12. Garey KW, Rege M, Pai MP, Mingo DE, Suda KJ, Turpin RS, Bearden DT: Time to initiation of fluconazole therapy impacts mortality in patients with candidemia: a multi-institutional study. Clin Infect Dis 2006, 43:25-31.

13. Kollef MH, Napolitano LM, Solomkin JS, Wunderink RG, Bae IG, Fowler VG, Balk RA, Stevens DL, Rahal JJ, Shorr AF, Linden PK, Micek ST: Health care-associated infection (HAI): a critical appraisal of the emerging threat-proceedings of the HAI Summit. Clin Infect Dis 2008, 47(Suppl 2):S55-99. quiz S100-101.

14. Kollef MH, Shorr A, Tabak YP, Gupta V, Liu LZ, Johannes RS: Epidemiology and outcomes of health-care-associated pneumonia: results from a large US database of culture-positive pneumonia. Chest 2005, 128:3854-3862.

15. Shorr AF, Tabak YP, Killian AD, Gupta V, Liu LZ, Kollef MH: Healthcare-associated bloodstream infection: A distinct entity? Insights from a large U.S. database. Crit Care Med 2006, 34:2588-2595.

16. Pappas PG: Invasive candidiasis. Infect Dis Clin North Am 2006, 20:485-506.

17. K:dzierska A, Kochan P, Pietrzyk A, K:dzierska J: Current status of fungal cell wall components in the immunodiagnostics of invasive fungal infections in humans: galactomannan, mannan and (1-->3)-beta-D-glucan antigens. Eur J Clin Microbiol Infect Dis 2007, 26:755-766.

18. lezzoni LI, Moskowitz MA: A clinical assessment of MedisGroups. JAMA 1988, 260:3159-3163.

19. Fine MJ, Auble TE, Yealy DM, Hanusa BH, Weissfeld LA, Singer $\mathrm{DE}$, Coley CM, Marrie TJ, Kapoor WN: A prediction rule to identify low-risk patients with community-acquired pneumonia. $N$ Engl J Med 1997, 336:243-250.

20. Shorr AF, Tabak YP, Gupta V, Johannes RS, Liu LZ, Kollef MH: Morbidity and cost burden of methicillin-resistant Staphylococcus aureus in early onset ventilator-associated pneumonia. Crit Care 2006, 10:R97.

21. Silber JH, Rosenbaum PR, Schwartz JS, Ross RN, Williams SV: Evaluation of the complication rate as a measure of quality of care in coronary artery bypass graft surgery. JAMA 1995, 274:317-323.

22. Tabak YP, Johannes RS, Silber JH: Using automated clinical data for risk adjustment: development and validation of six disease-specific mortality predictive models for pay-for-performance. Med Care 2007, 45:789-805.

23. Therneau TM, Atkinson EJ: An introduction to recursive partitioning using the RPART routines. Technical Report 61. Mayo Clinic, Section of Statistics 1997, 61: [http://www.mayo.edu/hsr/ techrpt/61.pdf]. Accessed December 31, 2008:1-52.

24. Breiman L, Friedman JH, Olshen RA, Stone CJ: Classification and regression trees. Belmont, CA: Wadsworth International Group; 1984.

25. Goldman L, Cook EF, Johnson PA, Brand DA, Rouan GW, Lee TH: Prediction of the need for intensive care in patients who come to the emergency departments with acute chest pain. N Engl $J$ Med 1996, 334:1498-1504.

26. Fonarow GC, Adams KF Jr, Abraham WT, Yancy CW, Boscardin WJ: Risk stratification for in-hospital mortality in acutely decompensated heart failure: classification and regression tree analysis. JAMA 2005, 293:572-580.

27. Aujesky D, Obrosky DS, Stone RA, Auble TE, Perrier A, Cornuz J, Roy PM, Fine MJ: A prediction rule to identify low-risk patients with pulmonary embolism. Arch Intern Med 2006, 166:169-175.

28. Takahashi O, Cook EF, Nakamura T, Saito J, lkawa F, Fukui T: Risk stratification for in-hospital mortality in spontaneous intracerebral haemorrhage: a Classification and Regression Tree analysis. Ojm 2006, 99:743-750. 
29. Breslow NE, Day NE: Statistical methods in cancer research. Volume I - The analysis of case-control studies. IARC Sci Publ 1980:5-338.

30. León C, Ruiz-Santana S, Saavedra P, Almirante B, Nolla-Salas J, Alvarez-Lerma F, Garnacho-Montero J, León MA: A bedside scoring system ("Candida score") for early antifungal treatment in nonneutropenic critically ill patients with Candida colonization. Crit Care Med 2006, 34:730-737.

31. Ostrosky-Zeichner L, Sable C, Sobel J, Alexander BD, Donowitz G, Kan V, Kauffman CA, Kett D, Larsen RA, Morrison V, Nucci M, Pappas PG, Bradley ME, Major S, Zimmer L, Wallace D, Dismukes WE, Rex JH: Multicenter retrospective development and validation of a clinical prediction rule for nosocomial invasive candidiasis in the intensive care setting. Eur J Clin Microbiol Infect Dis 2007, 26:271-276.

32. Michalopoulos AS, Geroulanos S, Mentzelopoulos SD: Determinants of candidemia and candidemia-related death in cardiothoracic ICU patients. Chest 2003, 124:2244-2255.

33. Amrutkar PP, Rege MD, Chen H, LaRocco MT, Gentry LO, Garey $\mathrm{KW}$ : Comparison of risk factors for candidemia versus bacteremia in hospitalized patients. Infection 2006, 34:322-327.

34. Labelle AJ, Micek ST, Roubinian N, Kollef MH: Treatment-related risk factors for hospital mortality in Candida bloodstream infections. Crit Care Med 2008, 36:2967-2972.

35. Blumberg HM, Jarvis WR, Soucie JM, Edwards JE, Patterson JE, Pfaller MA, Rangel-Frausto MS, Rinaldi MG, Saiman L, Wiblin RT, Wenzel RP: Risk factors for candidal bloodstream infections in surgical intensive care unit patients: the NEMIS prospective multicenter study. The National Epidemiology of Mycosis Survey. Clin Infect Dis 2001, 33:177-186. 